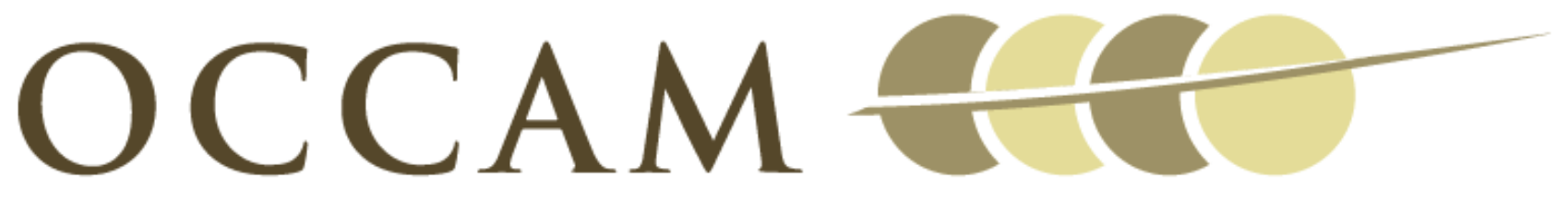

OXFORD CENTRE FOR COLLABORATIVE APPLIED MATHEMATICS

\author{
Report Number 11/10
}

On the predictions and limitations of the BeckerDoring model for reaction kinetics in micellar surfactant solutions

by

I. M. Griffiths, C. D. Bain, C. J. W. Breward, D. M. Colegate, P. D. Howell, S. L. Waters

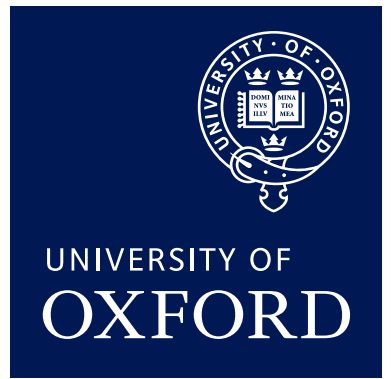

Oxford Centre for Collaborative Applied Mathematics Mathematical Institute 24 - 29 St Giles'

Oxford

OX1 3LB

England 



\title{
On the predictions and limitations of the Becker-Döring model for reaction kinetics in micellar surfactant solutions
}

\author{
I. M. Griffiths ${ }^{\mathrm{a}, *}$, C. D. Bain ${ }^{\mathrm{b}}$, C. J. W. Breward ${ }^{\mathrm{a}}$, D. M. Colegate ${ }^{\mathrm{b}}$, \\ P. D. Howell ${ }^{\mathrm{a}}$, S. L. Waters ${ }^{\mathrm{a}}$ \\ ${ }^{a}$ Mathematical Institute, University of Oxford, OX1 3LB. \\ ${ }^{b}$ Department of Chemistry, Durham University, DH1 3LE.
}

\begin{abstract}
We investigate the breakdown of a system of micellar aggregates in a surfactant solution following an order-one dilution. We derive a mathematical model based on the Becker-Döring system of equations, using realistic expressions for the reaction constants fit to Molecular Dynamics simulations. We exploit the largeness of typical aggregation numbers to derive a continuum model, substituting a large system of ordinary differential equations for a partial differential equation in two independent variables: time and aggregate size. Numerical solutions demonstrate that re-equilibration occurs in two distinct stages over well-separated time-scales, in agreement with experiment and with previous theories. We conclude by exposing a limitation in the Becker-Döring theory for re-equilibration and discuss potential resolutions.
\end{abstract}

Keywords: surfactant; micellization; aggregation kinetics.

\section{Introduction}

A surfactant is a chemical that preferentially accumulates at interfaces. Surfactants in water have both hydrophilic and hydrophobic groups. At the surface of water, the individual molecules (monomers) orient themselves so that the hydrophobic group protrudes from the water, lowering the surface tension. As the bulk surfactant concentration is increased, the amount of adsorbed surfactant increases until it becomes energetically more favourable for monomers to combine within the bulk and form aggregates that orient

\footnotetext{
*Corresponding author

Email address: ian.griffiths@maths.ox.ac.uk (I. M. Griffiths)
} 
their hydrophobic components towards the centre of the cluster. These aggregates can have various sizes and shapes but for many simple surfactants with a single hydrocarbon chain the aggregates are approximately spherical and contain of the order of 100 monomers [10]. The distribution of aggregate sizes is localized around this optimum value with a half-width of the order of the square root of the aggregation number. Aggregates that are much smaller than the mean aggregation number are energetically highly unfavourable and consequently appear in much lower concentrations [1, 2, 3, 27]. Clusters around the optimum aggregation number are called micelles, and the bulk surfactant concentration at which micelles first form is called the critical micelle concentration (CMC). The CMC is well-defined for a given surfactant at a given temperature and a common empirical means of determining its value is by determining the bulk concentration above which the surface tension is constant.

The re-equilibration of a surfactant solution upon a disturbance from equilibrium is of great importance for the adsorption kinetics of micellar solutions. Much of the experimental and theoretical work aimed at understanding dynamic systems of micelles was performed in the 1960s and 1970s, with Aniansson and Wall [1, 2, 3] producing the generally accepted theoretical description for the available kinetic data. The key assumption of this theory is that the breakdown and formation of aggregates occurs via stepwise monomer loss and gain. This leads to the Becker-Döring description, first introduced by Becker and Döring in 1935 [5].

The Becker-Döring equations are a special case of Smoluchowski coagulation theory [33] which, more generally, allows all aggregates sizes to combine and dissociate. Coagulation theory has been used to model aggregation in numerous situations. (For a review see [37] and references therein.) The original Becker-Döring formulation describes a system in which the monomer concentration is held constant. This can be interpreted as a phase transition in which a supersaturated gas condenses to form liquid drops at constant pressure. Penrose and Lebowitz [29] extend this theory to account for systems which conserve mass. Billingham and Coveney [7] consider the formation of micelles in a system out of thermodynamic equilibrium, and a reduced description of this system which preserves all the properties of the infinite-dimensional Becker-Döring equations is presented by Coveney and Wattis [12]. Coagulation theory has also been analysed in more complex situations, such as within a flowing fluid, with particular application to biological systems. For example, Band et al. [4] combine the Becker-Döring theory with an advection-diffusion model to describe crystal aggregation in the lower urinary tract, while Guy et al. [15] model the formation of a blood clot in a shearing flow. 
In almost all situations considered, either the monomer concentration is held constant, or the aggregates are assumed to have a uniform concentration distribution, both of which simplify the analysis significantly. Billingham [6] relaxes the latter assumption when applying the Becker-Döring theory to the coagulation of monomers choosing rate constants that produce realistic equilibrium aggregate distributions, and determines asymptotic solutions for the formation of micelles upon a slow influx of monomer into a solution. Aniansson and Wall [1] consider a realistic aggregation distribution comprised predominantly of either monomers or aggregates localized around the large optimum aggregation number, with aggregates in between occurring at much lower concentrations. However, they make the crucial assumption that the deviation from equilibrium is sufficiently small to derive a set of linearized Becker-Döring equations describing the variations in aggregate concentrations from their equilibrium values. Their model predicts that the relaxation occurs in two stages on well-separated timescales, which are inherently related to the shape of the aggregate distribution.

The existence of a two-timescale re-equilibration process was verified experimentally by Lang et al. [22]. The first, more rapid, timescale corresponds to the replenishment of monomer via release of individual monomers from aggregates. However, to return the monomer to its equilibrium value requires some aggregates to break down entirely. Some of the monomers released replenish the monomer concentration to its critical value, while the remainder join those aggregates which have not broken down. The associated relaxation times differ by at least three orders of magnitude, with monomer loss occurring on the $\mu \mathrm{s}-\mathrm{ms}$ timescale and complete micelle breakdown on the msminutes timescale [10]. Aniansson et al. [3] showed how their model could be used to fit Lang's experimental data and extract information about the aggregate distribution, and the theory has since been applied by numerous experimentalists $[8,14,17,23,24,28,39]$.

Aniansson and Wall's model is still the cornerstone for the analysis of micellar kinetic data, with the theory on micelle exchange kinetics remaining essentially unchanged. Recently, Rusanov et al. [20, 18, 19, 21, 32] provided mathematical analysis of the micellization process, based on the Aniansson and Wall kinetic model. Their theory outlines no fewer than nine characteristic kinetic times of micellization in non-ionic surfactant solutions, although they propose no method of probing these experimentally.

While some temperature-jump and pressure-jump experiments do indeed satisfy the limit of small disturbances from equilibrium examined by Aniansson and Wall, there are many physically important situations in which deviations from equilibrium are not small. Large-deviation re-equilibration has been well studied only in scenarios where there are a finite number of 
aggregates, all of which occur in equal concentrations at equilibrium (for example [4, 37]). However, the re-equilibration of a surfactant with a realistic aggregate distribution following an order-one dilution has not been analysed in detail. As a consequence, the Aniansson and Wall model has been applied to many situations where the deviations from equilibrium are much too large for the linearized theory to be applicable. In this paper we use the full Becker-Döring model to investigate the relaxation upon an order-one dilution (which leaves the system above the CMC) of a micellar surfactant solution with a realistic equilibrium aggregate distribution. In this case, the monomer concentration must be replenished to its equilibrium value via the breakdown of some of the aggregates, while a proportion of surfactant will still reside in aggregate form. We describe the mechanism by which this is achieved and the restructuring process of aggregates that ensues, demonstrating that the two-timescale behaviour predicted by Aniansson and Wall for small deviations from equilibrium is still a prominent feature.

Richardson et al. [31] exploit the large number of monomers that typically comprise an aggregate to derive a continuum model for the formation of lipid/protein microdomain structures within plasma membranes which interact via Smoluchowski coagulation theory. Tracking the evolution of a continuous function rather than the concentration of all individual species vastly simplifies the problem, and we employ a similar strategy in this paper.

We first validate our continuum model by comparing its predictions with simulations of the discrete Becker-Döring system. We then use this representation to elucidate the two-timescale behaviour and to analyse the two stages of re-equilibration. We show that the relative concentration of smaller aggregates to the micelle concentration is a key parameter, setting the relative timescales at which the two processes occur.

The predictions of our models are compared with experimental data obtained from stopped-flow experiments [10]. Here, surfactant solution is rapidly mixed with water by being forced into a mixing chamber and then into an observation cell. When the flow is stopped the reaction is monitored by using fluorescence techniques to measure the spectroscopic response with time.

We conclude by discussing surfactant systems for which our description fails. In such circumstances the assumptions made by the Becker-Döring theory must be relaxed, and a new mechanism for micelle breakdown must exist, which we analyse in a follow-up to this paper. 


\section{A discrete model}

\subsection{The Becker-Döring equations}

As discussed in the Introduction, the self-assembly and dissociation of aggregates is assumed to occur via stepwise monomer loss and gain [1, 2, 3], in the following reaction scheme:

$$
\text { monomer }+n \text {-mer } \underset{\kappa_{n}^{-}}{\stackrel{\kappa_{n}^{+}}{\rightleftharpoons}}(n+1) \text {-mer. }
$$

Here, we use the term $n$-mer to denote an aggregate containing $n$ monomers, and $\kappa_{n}^{ \pm}$are the association and dissociation rate coefficients. The reaction kinetics for this system are described by the Becker-Döring equations [5]

$$
\frac{\mathrm{d} \mathcal{X}_{n}}{\mathrm{~d} \mathcal{T}}=\kappa_{n-1}^{+} \mathcal{X}_{1} \mathcal{X}_{n-1}-\kappa_{n-1}^{-} \mathcal{X}_{n}-\kappa_{n}^{+} \mathcal{X}_{1} \mathcal{X}_{n}+\kappa_{n}^{-} \mathcal{X}_{n+1},
$$

for $n \geq 2$, where $\mathcal{X}_{n}=\mathcal{X}_{n}(\mathcal{T})$ denotes the (molar) concentration of an aggregate containing $n$ monomers at time $\mathcal{T}$.

The net bulk concentration of monomer contained in all aggregates is given by

$$
\mathcal{C}_{b}=\sum_{n=1}^{\infty} n \mathcal{X}_{n}
$$

Under the assumption that $\mathcal{C}_{b}$ is conserved for all time, the free monomer concentration is determined by

$$
\mathcal{X}_{1}(t)=\mathcal{C}_{b}-\sum_{n=2}^{\infty} n \mathcal{X}_{n}(t)=\mathcal{X}_{1}(0)-\sum_{n=2}^{\infty} n\left(\mathcal{X}_{n}(t)-\mathcal{X}_{n}(0)\right)
$$

Along with (2), this gives us an infinite-dimensional system of ODEs for $\mathcal{X}_{2}(t), \mathcal{X}_{3}(t), \cdots$. The solution of this system requires us to specify all the initial concentrations $\mathcal{X}_{1}(0), \mathcal{X}_{2}(0), \ldots$

For mathematical simplicity, it is customary to truncate the system at some large finite value $n=N$ and to assume that all the reaction rates are equal (see, for example, [36]). This is equivalent to setting

$$
\kappa_{n}^{ \pm}= \begin{cases}\kappa^{ \pm} & 1 \leq n \leq N-1 \\ 0 & n \geq N\end{cases}
$$

As we will see below, this approximation fails to capture the correct physics for many real-life systems, and we will focus on analysing the system (2), (4) with reaction rates consistent with real surfactants. 


\subsection{The equilibrium distribution}

Determination of the aggregate size distribution for different surfactants and different micelle shapes is a subject of extensive debate. Since concentrations in the intermediate aggregate region are orders of magnitude smaller than those close to the optimum aggregation number, there are no direct experimental methods available for their measurement. It is, however, possible to calculate an equilibrium aggregate size distribution from knowledge of the chemical potential differences between monomers in different sized aggregates. This may be done via Molecular Dynamics (MD) simulations $[13,16,25]$ or by Molecular Thermodynamics (MT) [26, 27, 30]. Either method predicts a distribution characterized by the following key features. Almost all surfactant material is contained within either a region of pre-micellar aggregates (monomer, dimers, trimers etc) or a region of proper micelles close to the peak aggregation number. These are connected by an intermediate region containing a very low concentration of aggregates; see figure 1. (The change in slope at $n=90$ arises from a change in the model from a sphere to a rod with spherical end-caps. In reality, micelles will pass through an ellipsoidal shape between spheres and rods that smooths out the distribution.) These predictions are in agreement with experimental techniques such as light-scattering methods or small-angle neutron scattering (SANS) experiments which may be used to determine the optimum aggregation number, and stopped-flow experiments to determine the CMC [10].

In modelling terms, an equilibrium distribution corresponds to a steady solution of the Becker-Döring system. Substitution of $\mathcal{X}_{n}=\mathcal{X}_{n}^{*}=$ constant into (2), (4) yields a system of algebraic equations to determine $\mathcal{X}_{n}^{*}$, in terms of the reaction coefficients $\kappa_{n}^{ \pm}$and the net surfactant concentration $\mathcal{C}_{b}$. At equilibrium, the principle of microscopic reversibility requires that each mechanistic step in a reversible reaction must itself be in equilibrium, and so equation (2) implies that

$$
\kappa_{n}^{+} \mathcal{X}_{1}^{*} \mathcal{X}_{n}^{*}-\kappa_{n}^{-} \mathcal{X}_{n+1}^{*} \equiv \kappa_{i}^{+} \mathcal{X}_{1}^{*} \mathcal{X}_{i}^{*}+\kappa_{i}^{-} \mathcal{X}_{i+1}^{*},
$$

for all $n, i \geq 1$. To ensure that the system contains a finite amount of surfactant, we must have $\mathcal{X}_{i}^{*} \rightarrow 0$ as $i \rightarrow \infty$, and hence

$$
\kappa_{n}^{+} \mathcal{X}_{1}^{*} \mathcal{X}_{n}^{*} \equiv \kappa_{n}^{-} \mathcal{X}_{n+1}^{*}
$$

for all $n$. Given the reaction coefficients, (7) determines the equilibrium distribution

$$
\mathcal{X}_{n}^{*}=\mathcal{X}_{1}^{* n} \prod_{i=1}^{n-1}\left(\frac{\kappa_{i}^{+}}{\kappa_{i}^{-}}\right)
$$




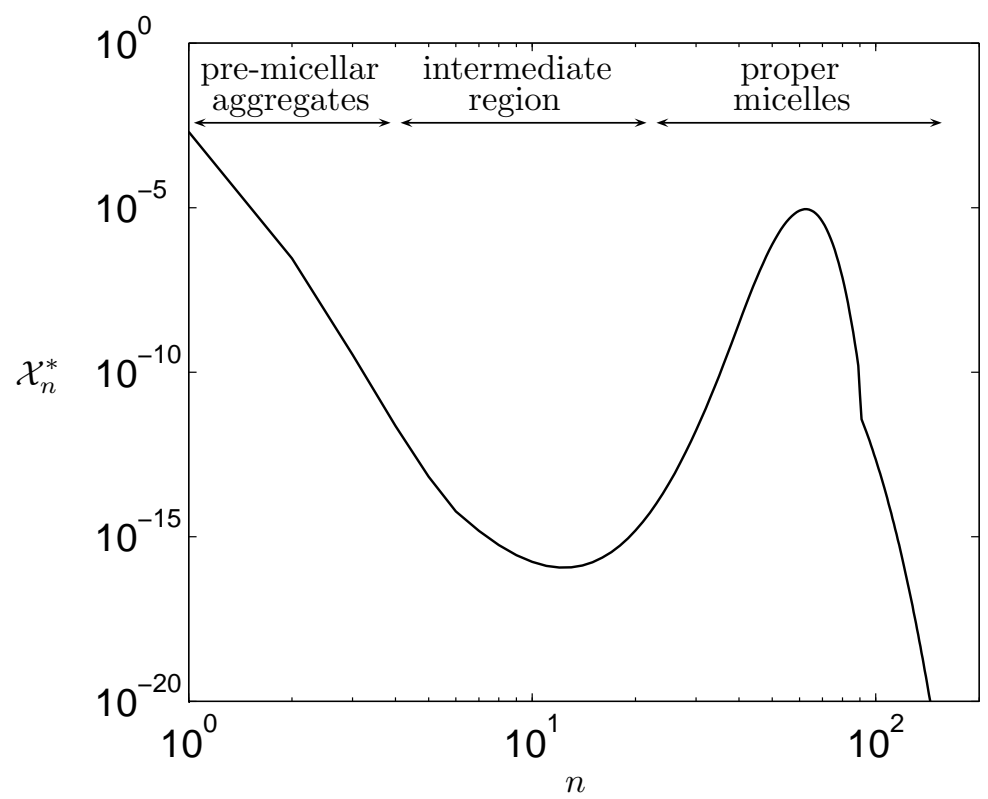

Figure 1: An equilibrium aggregate size distribution predicted by MD simulation for the polyoxyethylene glycol alkyl ether surfactant $\mathrm{C}_{10} \mathrm{E}_{8}$ at a bulk concentration $C_{b}=$ $10 \mathrm{mM}[10]$.

in terms of the monomer concentration $\mathcal{X}_{1}^{*}$, which is related to the net concentration $\mathcal{C}_{b}$ by

$$
\mathcal{C}_{b}=\sum_{n=1}^{\infty} n \mathcal{X}_{1}^{* n} \prod_{i=1}^{n-1}\left(\frac{\kappa_{i}^{+}}{\kappa_{i}^{-}}\right)
$$

In practice, the reaction rates are not well characterized. Instead, we suppose that the equilibrium distribution itself is known, for some particular bulk concentration $\mathcal{C}_{b}$, either from MD or from MT, and takes a form similar to that shown in figure 1 . We can then use (7) to relate the reaction rates:

$$
\kappa_{n}^{-}=\left(\frac{\mathcal{X}_{1}^{*} \mathcal{X}_{n}^{*}}{\mathcal{X}_{n+1}^{*}}\right) \kappa_{n}^{+}
$$

for $n \geq 1$, which enforces the principle of microscopic reversibility.

For the association rates, ultrasonic adsorption studies [34, 38] suggest that reactions proceed at a diffusion-controlled rate, so that

$$
\kappa_{n}^{+} \propto N_{A} \sigma_{n}\left(D_{1}+D_{n}\right),
$$

where $N_{A}$ is the Avogadro number, $D_{n}$ represents the diffusion coefficient of an aggregate of size $n$, and $\sigma_{n}$ is the collision radius. We may take $\sigma_{n}$ to be 
the sum of the aggregate radii, $r_{1}+r_{n}$ (with $r_{n}$ proportional to $n^{1 / 3}$ ), and the Stokes-Einstein equation provides a relation between the diffusion coefficient of an aggregate and its size, namely

$$
D_{n}=\frac{k_{B} T}{6 \pi \mu r_{n}}
$$

where $k_{B}$ is the Boltzmann constant, and $T$ and $\mu$ are the temperature and viscosity of the solution. This provides the estimate

$$
\kappa_{n}^{+} \sim\left(\frac{k_{B} N_{A} T}{6 \pi \mu}\right) \frac{\left(1+n^{1 / 3}\right)^{2}}{n^{1 / 3}},
$$

which gives $\kappa_{1}^{+} \sim 6 \times 10^{5} \mathrm{~m}^{3} \mathrm{~mol}^{-1} \mathrm{~s}^{-1}$ for a surfactant in water at $300 \mathrm{~K}$. For the polyoxyethylene glycol alkyl ether surfactants $\mathrm{C}_{6} \mathrm{E}_{3}$ and $\mathrm{C}_{8} \mathrm{E}_{6}$, with CMCs of order $100 \mathrm{mM}$ and $12 \mathrm{mM}[11,35]$, equation (13) predicts reaction timescales of order $10^{-5} \mathrm{~s}$ and $10^{-4} \mathrm{~s}$ respectively. These are in line with spectroscopic stopped-flow experiments on the $\mathrm{C}_{n} \mathrm{E}_{m}$ series, where rapid reequilibration is observed to occur before data acquisition begins, that is, on a timescale less than $10 \mathrm{~ms}$ [10].

We note that the estimate (13) predicts rather weak dependence of the association rates upon aggregate size; for example $\kappa_{200}^{+}$is roughly twice $\kappa_{1}^{+}$. In contrast, we will see that the dissociation rates $\kappa_{n}^{-}$vary significantly with $n$. As a first approximation, we will therefore suppose henceforth in this paper that the association rates are all equal. Given an equilibrium distribution $\mathcal{X}_{n}^{*}$, we may thus use (10) to determine all the reaction rates

$$
\kappa_{n}^{+}=\kappa_{1}^{+}, \quad \kappa_{n}^{-}=\left(\frac{\mathcal{X}_{1}^{*} \mathcal{X}_{n}^{*}}{\mathcal{X}_{n+1}^{*}}\right) \kappa_{1}^{+},
$$

for $n=2,3,4, \ldots$, in terms of a single rate constant $\kappa_{1}^{+}$.

The continuum model we derive in $\S 3$ is valid for any equilibrium distribution $\mathcal{X}_{n}^{*}$ resembling that shown in figure 1 , with surfactant concentrated in narrow neighbourhoods of $n=1$ and $n=m$ (the peak aggregation number), separated by an intermediate region of very low concentration. However, we will find it helpful to fix ideas by focusing on a particular fit to the equilibrium distribution, namely

$$
\frac{\mathcal{X}_{n}^{*}}{\mathcal{X}_{1}^{*}}=A \exp (b-b n)+\frac{B}{m^{3 / 2}} \exp \left(-\beta m(n / m-1)^{2}\right)+\frac{C}{m^{2}} \exp (-d n / m),
$$

where $A, B, C, b, \beta, d$ and $m$ are fitting parameters. The first term represents the spike at $n=1$ and the small number of pre-micellar aggregates. 
The second term captures the distribution of proper micelles with a width determined by $\beta$. The third term is a slowly decaying exponential to represent the concentration of intermediate aggregates: the value of $C$ can be used to control the ratio of maximum to minimum aggregate concentrations. The scaling of the second and third terms by $m^{-3 / 2}$ and $m^{-2}$, respectively, ensures that the importance of these terms relative to each other and to the monomer concentration depends on the coefficients $A, B$ and $C$ and not on the most probable aggregation number, $m$. The distribution (15) captures the key features predicted by MT illustrated in figure 1. In particular, the largeness of $m$ ensures the high relative concentration of monomer to aggregates, as well as a narrow Gaussian spread around $n=m$.

We can fix two parameters, for example $A$ and $B$, by requiring the righthand side of (15) to be equal to 1 when $n=1$ and by specifying the net concentration:

$$
\begin{gathered}
1=A+\frac{B}{m^{3 / 2}} \mathrm{e}^{-\beta(m-1)^{2} / m}+\frac{C}{m^{2}} \mathrm{e}^{-d / m}, \\
C_{b}=\frac{\mathcal{C}_{b}}{\mathcal{X}_{1}^{*}}=\frac{A}{\left(1-\mathrm{e}^{-b}\right)^{2}}+\frac{B}{m^{3 / 2}} \sum_{n=1}^{\infty} n \mathrm{e}^{-\beta m(n / m-1)^{2}}+\frac{C \mathrm{e}^{-d / m}}{m^{2}\left(1-\mathrm{e}^{-d / m}\right)^{2}} .
\end{gathered}
$$

Here we have introduced for convenience the dimensionless bulk concentration $C_{b}$, scaled with the equilibrium monomer concentration $\mathcal{X}_{1}^{*}$. In figure 2 , we show a typical MD prediction for the surfactant $\mathrm{C}_{6} \mathrm{E}_{3}$ alongside our ansatz (15), with chosen parameter values $b=4.7, \beta=0.765, C=3.47 \times 10^{-4}$, $d=2.72, C_{b}=10$ and $m=34$ from which we infer that $A \approx 1$ and $B \approx 2.26$.

For many realistic surfactants, the concentration of aggregates in the intermediate region is extremely low, which corresponds to very small values of $C$. As we will find, this leads to extreme separation of timescales in the re-equilibration process: rapid disassociation via stepwise monomer release followed by excruciatingly slow micelle reassembly to reach the equilibrium. To begin with, we shall consider systems for which $C$ is not too small, which serves to illustrate the behaviour without compromising computational expediency.

\subsection{The post-dilution distribution}

We now consider a surfactant system that starts in equilibrium with a known distribution, $\mathcal{X}_{n}=\mathcal{X}_{n}^{*}$, before being instantaneously diluted. Upon dilution of the system by a factor $D>1$, the initial distribution $\mathcal{X}_{n}(0)$ becomes $\mathcal{X}_{n}^{*} / D$, and the new equilibrium distribution, say $\overline{\mathcal{X}}_{n}$, satisfies

$$
\frac{\overline{\mathcal{X}}_{1} \overline{\mathcal{X}}_{n}}{\overline{\mathcal{X}}_{n+1}}=\frac{\mathcal{X}_{1}^{*} \mathcal{X}_{n}^{*}}{\mathcal{X}_{n+1}^{*}},
$$




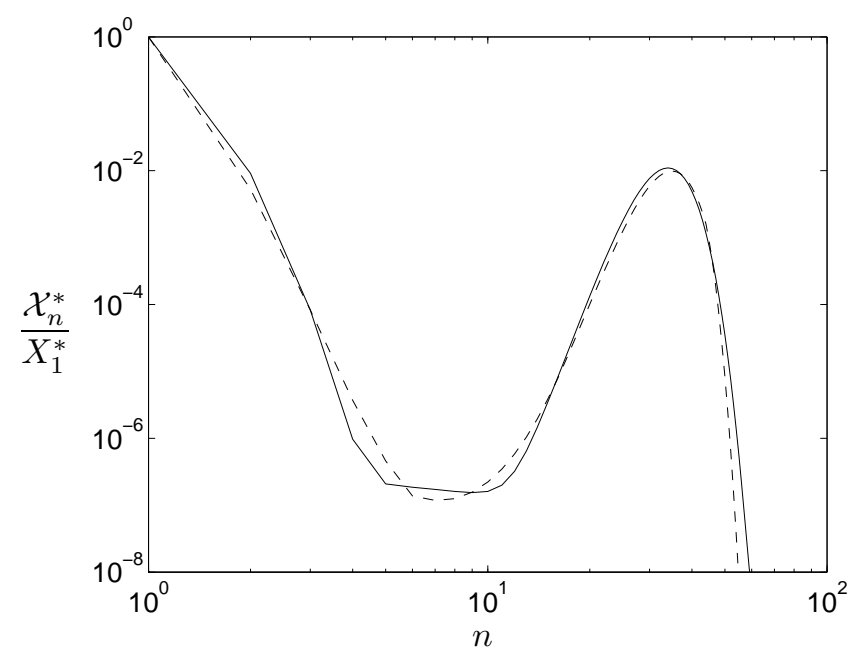

Figure 2: Comparison between equilibrium distribution for the surfactant $\mathrm{C}_{6} \mathrm{E}_{3}$ at a bulk concentration $C_{b}=10$, predicted by MT (dashed) and the ansatz (15) (solid) with $A=1$, $b=4.7, B=2.26, \beta=0.765, m=34, C=3.47 \times 10^{-4}, d=2.72[9]$.

for $n \geq 1$, from (2) and (10), assuming that the rate constants $\kappa_{n}^{ \pm}$are unaffected by a dilution. Equation (18) may be used recursively to give

$$
\overline{\mathcal{X}}_{n}=\nu^{n} \mathcal{X}_{n}^{*}
$$

for $n \geq 1$, where $\nu$ is determined implicitly by

$$
\sum_{n=1}^{\infty} n \mathcal{X}_{n}^{*}\left(1-D \nu^{n}\right)=0
$$

which enforces global conservation of surfactant.

In figure 3 we plot the dependence of $\nu$ on the dilution factor $D$ for the equilibrium distribution (15) with illustrative parameter values $b=10$, $C=0.5, d=1, \beta=0.2, m=100$ and $C_{b}=10$, from which we deduce that $A \approx 1$ and $B \approx 2.27$. Provided we dilute by a factor $D<C_{b}$ so that there remains sufficient surfactant to replenish the depleted monomer to its critical value, then $\nu$ remains close to 1 . In this case, the equilibrium monomer concentration $\overline{\mathcal{X}}_{1}$ after dilution is only slightly smaller than the pre-dilution equilibrium concentration $\mathcal{X}_{1}^{*}$. On the other hand, when the solution is diluted so that the total bulk concentration is below $\mathcal{X}_{1}^{*}$, there is insufficient surfactant in the form of aggregates to replenish the monomer to the CMC, and $\nu$ then decreases significantly. In figure 3 this is clearly evident upon dilution by a factor $D>C_{b}=10$ in this case. These observations reinforce the 


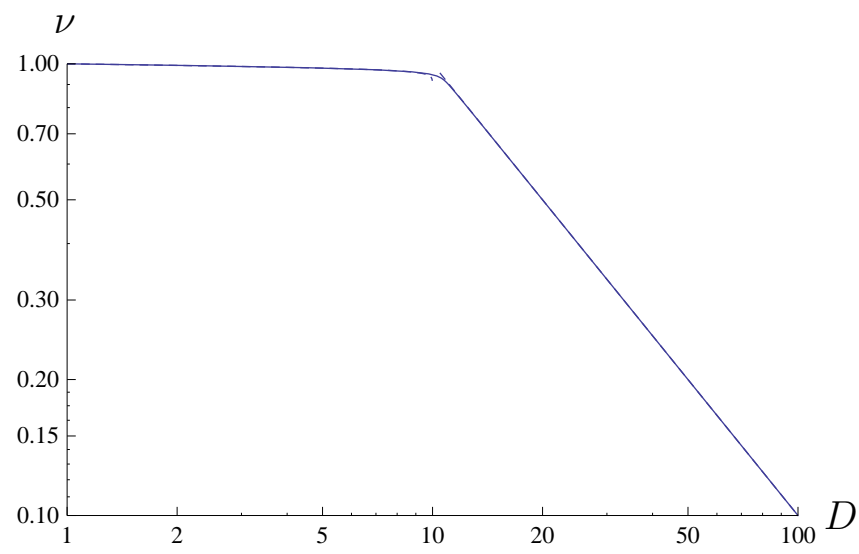

Figure 3: The dependence of the parameter $\nu$ on the dilution ratio $D$ for the equilibrium distribution (15) with parameter values $b=10, \beta=0.2, C=0.5, d=1, m=100$ and $C_{b}=10$. The dashed curves show the asymptotic prediction (21).

fact that, as surfactant is added to solution, it exists only in monomer form until the bulk concentration reaches a well-defined critical concentration (the $\mathrm{CMC}$ ), while further increases in bulk concentration increase the monomer concentration only marginally, so that the majority of the excess surfactant exists in aggregate form.

The distinctive form of the graph shown in figure 3 may be understood by analysing the implicit relation (20) asymptotically in the limit $m \rightarrow \infty$. The analysis outlined in $\S 3.2$ shows that $\nu$ and $D$ are related approximately by

$$
\nu \sim \begin{cases}\left(\frac{C_{b}-D}{\left(C_{b}-1\right) D}\right)^{1 / m} & D<C_{b}, \\ \frac{C_{b}}{D} & D>C_{b} .\end{cases}
$$

These are shown in figure 3 as dashed curves, which are virtually indistinguishable from the exact relation (20).

Using the formula (15) to fit the pre-dilution equilibrium distribution $\mathcal{X}_{n}^{*}$, we find that the initial and equilibrium states following dilution have exactly 
the same form, namely

$$
\begin{aligned}
\frac{\mathcal{X}_{n}(0)}{\overline{\mathcal{X}}_{1}} & =A_{0} \exp (b-b n)+\frac{B_{0}}{m^{3 / 2}} \exp \left(-\beta m(n / m-1)^{2}\right)+\frac{C_{0}}{m^{2}} \exp (-d n / m), \\
\frac{\overline{\mathcal{X}}_{n}}{\overline{\mathcal{X}}_{1}} & =A \exp (\bar{b}-\bar{b} n)+\frac{\bar{B}}{m^{3 / 2}} \exp \left(-\bar{\beta} \bar{m}(n / \bar{m}-1)^{2}\right)+\frac{\bar{C}}{m^{2}} \exp (-\bar{d} n / m),
\end{aligned}
$$

where

$$
\begin{gathered}
A_{0}=\frac{A}{\nu D}, \quad B_{0}=\frac{B}{\nu D}, \quad C_{0}=\frac{C}{\nu D}, \\
\bar{b}=b-\log \nu, \quad \bar{B}=B \nu^{m-1} \exp \left(\frac{m \log ^{2} \nu}{4 \beta}\right), \quad \bar{C}=\frac{C}{\nu}, \\
\bar{\beta}=\bar{\omega} \beta, \quad \bar{m}=\bar{\omega} m, \quad \bar{d}=d-m \log \nu,
\end{gathered}
$$

and

$$
\bar{\omega}=1+\frac{\log \nu}{2 \beta} .
$$

Equation (23) implies that the dilution has the effect of shifting the optimum aggregation number from $m$ to $\bar{m}$, given by $(24 f)$. We illustrate the dependence of the ratio $\bar{\omega}=\bar{m} / m$ on the dilution ratio $D$ in figure 4 , for the equilibrium distribution (15). The dashed curves show the leading-order approximations corresponding to (21) in the limit $m \rightarrow \infty$, namely

$$
\bar{\omega} \sim \begin{cases}1-\frac{1}{2 m \beta} \log \left(\frac{\left(C_{b}-1\right) D}{C_{b}-D}\right) & D<C_{b}, \\ 1-\frac{1}{2 \beta} \log \left(\frac{D}{C_{b}}\right) & D>C_{b} .\end{cases}
$$

So long as $D<C_{b}$, we observe that $1-\bar{\omega}=O(1 / m)$, where $m$ is chosen to be large. The peak in the distribution is thus shifted only very slightly to the left by the dilution. This result validates the common assumption that the optimum aggregation number in a micellar solution is constant for a given surfactant. However, when the dilution is increased above the value $C_{b}$, the optimum aggregate size falls rapidly, with surfactant predominantly residing in monomer form for finite dilution ratios $D \gtrsim \mathrm{e}^{2 \beta} C_{b}$. This models the break-up of aggregates into monomers as the net bulk concentration falls below the CMC. 


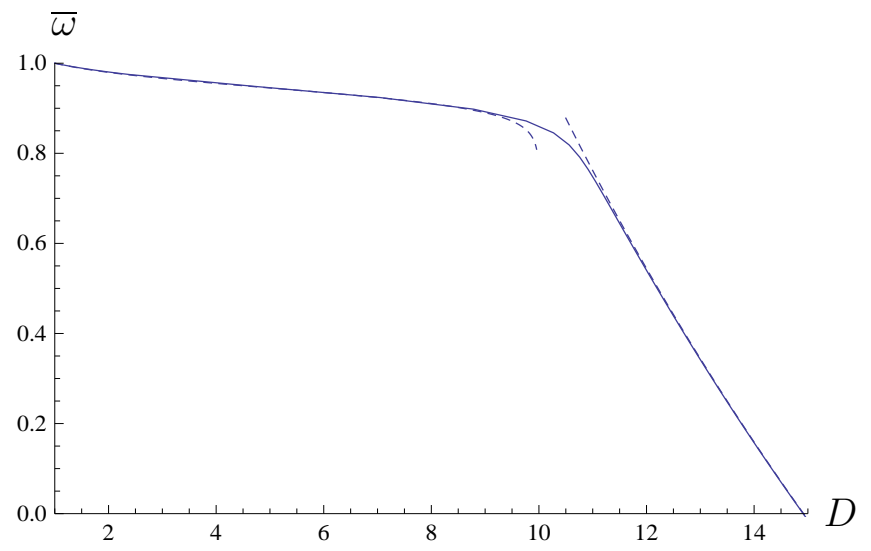

Figure 4: The dependence of the post-dilution peak aggregation number $\bar{\omega}$ on the dilution ratio $D$ for the equilibrium distribution (15) with parameter values $b=10, \beta=0.2$, $C=0.5, d=1, m=100$ and $C_{b}=10$. The dashed curves show the asymptotic prediction $(26)$.

\subsection{Non-dimensionalization and scaling}

We scale the concentrations following dilution with the equilibrium monomer post-dilution concentration $\overline{\mathcal{X}}_{1}=\nu \mathcal{X}_{1}^{*}$, while an appropriate timescale is set by the monomer association rate. We therefore define the dimensionless quantities $X_{n}, \bar{X}_{n}$ and $\tau$ as follows:

$$
\mathcal{X}_{n}=\overline{\mathcal{X}}_{1} X_{n} \quad \overline{\mathcal{X}}_{n}=\overline{\mathcal{X}}_{1} \bar{X}_{n}, \quad \mathcal{T}=\frac{\tau}{\kappa_{1}^{+} \overline{\mathcal{X}}_{1}} .
$$

The non-dimensional initial and final distributions are then given by

$$
X_{n}(0)=\frac{1}{\nu D} \frac{\mathcal{X}_{n}^{*}}{\mathcal{X}_{1}^{*}}, \quad \bar{X}_{n}=\nu^{n-1} \frac{\mathcal{X}_{n}^{*}}{\mathcal{X}_{1}^{*}},
$$

for $n \geq 1$. As described above, the parameter $\nu$ is determined from (20) for a given initial distribution $\mathcal{X}_{n}^{*}$ and dilution factor $D$.

The non-dimensional Becker-Döring equations (2), (4) may be written as

$$
\begin{aligned}
\frac{\mathrm{d} X_{n}}{\mathrm{~d} \tau} & =X_{1} X_{n-1}-\frac{\bar{X}_{n-1}}{\bar{X}_{n}} X_{n}-X_{1} X_{n}+\frac{\bar{X}_{n}}{\bar{X}_{n+1}} X_{n+1}, \\
X_{1} & =1-\sum_{n=2}^{\infty} n\left(X_{n}-\bar{X}_{n}\right),
\end{aligned}
$$

where we have used (14) to eliminate the rate constants $\kappa_{n}^{ \pm}$. Given the initial post-dilution distribution (28a), the system of equations (29) is used to determine the evolution towards the equilibrium $(28 b)$. 


\subsection{Numerical solutions}

In figure 5 we show the evolution of a typical distribution of aggregates, given by (15), with $b=10, \beta=0.2, C=0.5, d=1, m=100$ and $C_{b}=10$, upon a two-fold dilution. We solve $(20)$ to give $\nu \approx 0.992$ in this case, highlighting again the weak dependence of the equilibrium monomer concentration on the dilution factor provided the bulk concentration exceeds the CMC. We then solve the system (29) numerically.

As predicted for re-equilibration following a weak dilution $[1,2,3]$, the generic behaviour of the system is via a two-stage process. Firstly, the position of the micelle peak shifts to the left while retaining its magnitude, as aggregates shed individual monomers to replenish the concentration of monomers. This is called the $\tau_{1}$ process $[1,2,3]$. However, since the energetic stability of aggregates falls rapidly with decreasing aggregation number, this process becomes unfeasible before it has supplied enough material to replenish the monomer to its new equilibrium value. As a result, some of the aggregates have to break down completely to replenish the monomer fully to the new equilibrium. The remaining monomers associate with those aggregates which have not broken down to increase their typical aggregation number and hence their energetic stability. Thus the position of the peak in the aggregate distribution moves to the right and reduces in magnitude. This second process is called the $\tau_{2}$ process $[1,2,3]$. The $\tau_{2}$ process is governed by the rate at which micelles are able to break down entirely, which is set by the concentration of aggregates in the intermediate region: the lower the concentration of aggregates, the longer the time taken to re-equilibrate. These two distinct mechanisms are clearly illustrated in the graph of monomer concentration $X_{1}$ versus time in figure 6 . The monomer concentration initially rises rapidly, over a timescale of $0 \leq \tau \lesssim 1$ before the rate of replenishment slows, finally reaching equilibrium when $\tau \approx 50$.

\section{A continuum model}

\subsection{Introduction}

In figures 2 and 5 we exploited the largeness of the optimum aggregation number $m$ by joining the discrete set of values for each species concentration to form a smooth curve. Tracking the evolution of a single continuous function rather than the concentration of many aggregates provides a welcome simplification and invites us to seek a continuum limit to the Becker-Döring equations (29) in the limit $1 / m \rightarrow 0$. This approach both reduces the numerical complexity of the problem and uncovers analytical simplifications which provide deeper insight into the re-equilibration process. Richardson 


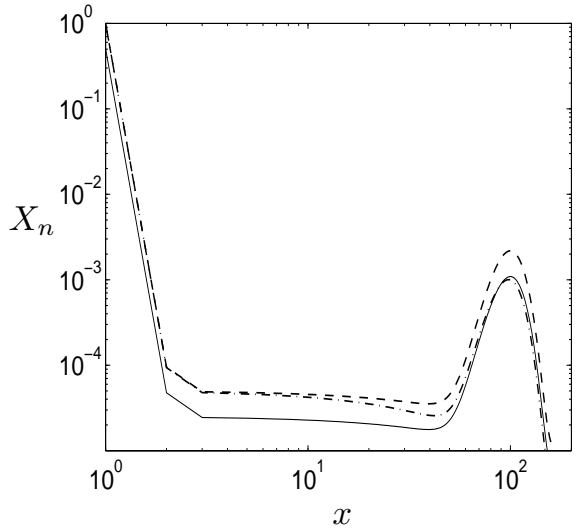

(a)

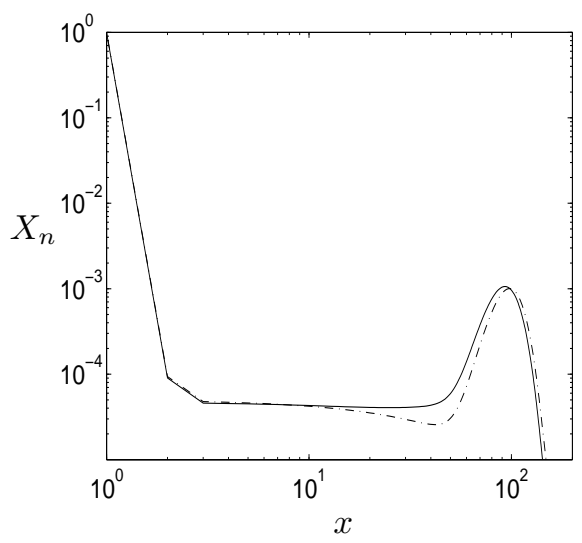

(c)

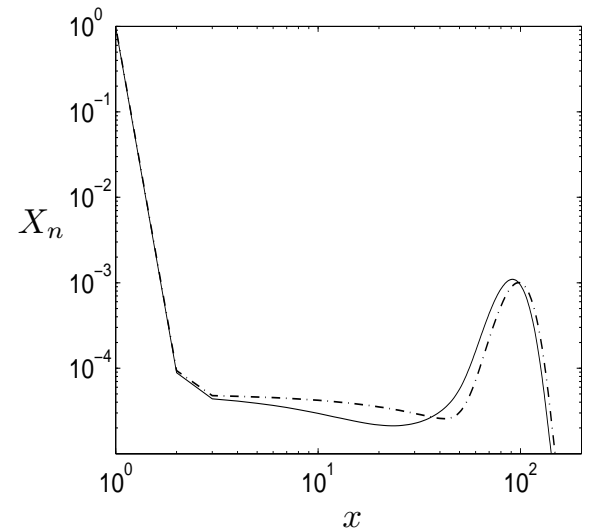

(b)

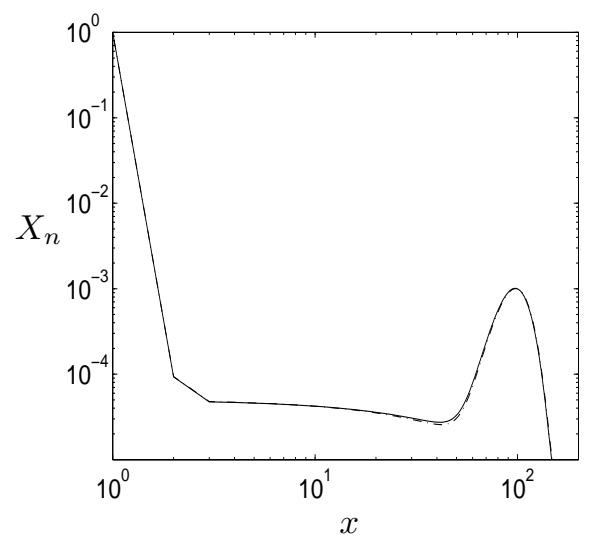

(d)

Figure 5: The solid curve shows the evolution of an initial pre-dilution distribution given by (15) with $b=10, \beta=0.2, C=0.5, d=1, m=100, C_{b}=10$ and $D=2$, at (a) $\tau=0$, (b) $\tau=100$, (c) $\tau=500$, (d) $\tau=5000$. The dot-dash line shows the equilibrium distribution. The pre-dilution distribution is shown as a dashed line in (a). 


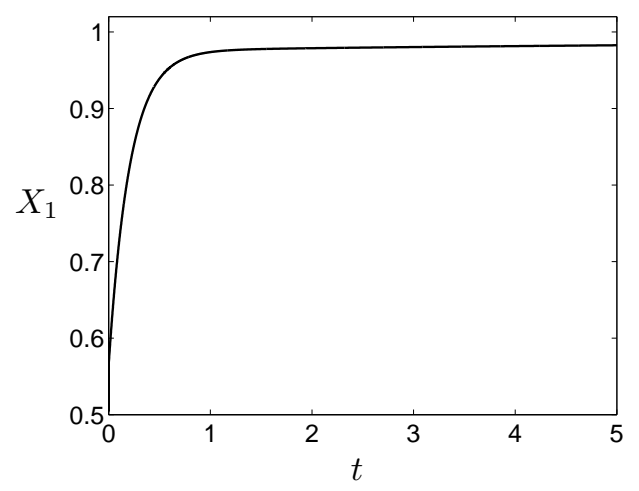

(a)

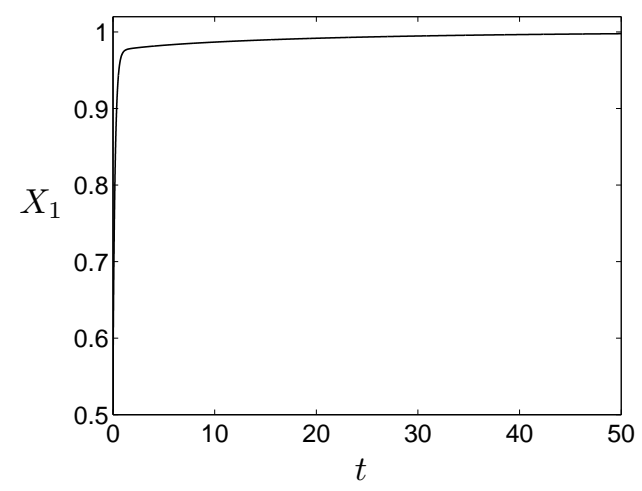

(b)

Figure 6: Monomer concentration $X_{1}(\tau)$ versus time for the pre-dilution distribution (15) with $b=10, \beta=0.2, C=0.5, d=1, m=100, C_{b}=10$ and $D=2$ on (a) the $\tau_{1}$ timescale, (b) the $\tau_{2}$ timescale.

et al. [31] utilize a similar approach to model the formation of lipid/protein microdomain structures within plasma membranes.

To implement our continuum model, we first exploit the fact that, in our distribution representation (15), the first exponential is solely responsible for the spike behaviour near $n=1$. We may therefore approximate the pre-dilution equilibrium distribution (15) as

$$
\frac{\mathcal{X}_{n}^{*}}{\mathcal{X}_{1}^{*}}= \begin{cases}1 & n=1 \\ \frac{B}{m^{3 / 2}} \exp \left(-\beta m(n / m-1)^{2}\right)+\frac{C}{m^{2}} \exp (-d n / m) & n \geq 2 .\end{cases}
$$

Outside a narrow neighbourhood of the optimal aggregation number $n=m$, the ansatz (15) indicates that the concentration scales with $\mathrm{m}^{2}$. We therefore choose to describe the pre-dilution equilibrium distribution of aggregates by the continuous order-one function $Y(n / m)=m^{2} \mathcal{X}_{n}^{*} / \mathcal{X}_{1}^{*}$ for $n \geq 2$, that is,

$$
Y(x)=B \sqrt{m} \exp \left(-\beta m(x-1)^{2}\right)+C \exp (-d x) .
$$

The post-dilution concentration is similarly scaled with $m^{2}$ by defining

$$
y(n / m, t)=m^{2} X_{n}(t)
$$

for $n \geq 2$. The initial and equilibrium post-dilution concentrations are then 
given by the continuous functions

$$
\begin{aligned}
y_{0}(x) & =y(x, 0)=\frac{Y(x)}{\nu D} \\
& =B_{0} \sqrt{m} \exp \left(-\beta m(x-1)^{2}\right)+C_{0} \exp (-d x), \\
\bar{y}(x) & =\lim _{t \rightarrow \infty} y(x, t)=Y(x) \nu^{m x-1} \\
& =\bar{B} \sqrt{m} \exp \left(-\beta m(x-\bar{\omega})^{2}\right)+\bar{C} \exp (-\bar{d} x),
\end{aligned}
$$

which replace (22) and (23) respectively, while the corresponding monomer concentrations are

$$
X_{1}(0)=\frac{1}{\nu D}, \quad \quad \lim _{t \rightarrow \infty} X_{1}(t)=\bar{X}_{1}=1 .
$$

The parameters in (33) are still related to the pre-dilution distribution by (24).

\subsection{Integral relations}

In the continuum limit, the non-dimensional pre-dilution bulk concentration $C_{b}$ is now expressed as an integral, namely

$$
C_{b}=1+\int_{0}^{\infty} x Y(x) \mathrm{d} x
$$

Using the fit (31) to $Y(x)$, we find that this integral may be evaluated to give

$$
C_{b}-1=B \sqrt{\frac{\pi}{\beta}} G(\sqrt{\beta m})+\frac{C}{d^{2}},
$$

where for convenience we introduce the function

$$
G(s)=\frac{1}{2}\left(\frac{\mathrm{e}^{-s^{2}}}{\sqrt{\pi} s}+\operatorname{erfc}(-s)\right) .
$$

We can use equation (36) to evaluate $B$ in terms of all the other fitting parameters. Since $G(\sqrt{\beta m}) \sim 1$ with exponential accuracy as $m \rightarrow \infty$, no further asymptotic error is incurred by using the approximation

$$
B \sim \sqrt{\frac{\beta}{\pi}}\left(C_{b}-1-\frac{C}{d^{2}}\right) .
$$

For example, with $\beta=0.2, m=100, C=0.5, d=1$, and $C_{b}=10$, we obtain $B \approx 2.27$; the value differs from that obtained using the discrete model in $\S 2.2$ by a factor of less than $10^{-5}$. 
The value of $\nu$ corresponding to a given dilution ratio $D$ can now be determined from the continuum version of (20), namely

$$
\frac{C_{b}}{\nu D}=1+\int_{0}^{\infty} x \bar{y}(x) \mathrm{d} x
$$

with $\bar{y}(x)$ given by (33b). Again, this integral may be calculated explicitly to give

$$
\frac{C_{b}}{D}-\nu=B \sqrt{\frac{\pi}{\beta}} \bar{\omega} \mathrm{e}^{-\beta m\left(1-\bar{\omega}^{2}\right)} G(\bar{\omega} \sqrt{\beta m})+\frac{C}{(d-m \log \nu)^{2}},
$$

where $\bar{\omega}$ is still defined by (25). The right-hand side of (40) is exponentially small unless $\nu$ and $\bar{\omega}$ are asymptotically close to 1 . We therefore obtain the leading-order relation

$$
\nu \sim \frac{C_{b}}{D}
$$

which is valid for $\nu<1$, that is, for $D>C_{b}$.

The right-hand side of (40) comes into play when

$$
\bar{\omega}=1-\frac{\kappa}{m \beta},
$$

so that $\nu=\mathrm{e}^{-2 \kappa / m}$, with $\kappa=O(1)$. In this case, equation (40) is approximated by

$$
\frac{C_{b}}{D}-\mathrm{e}^{-2 \kappa / m} \sim\left(C_{b}-1\right) \mathrm{e}^{-2 \kappa}\left(1-\frac{\kappa}{\beta m}\right) \mathrm{e}^{\kappa^{2} / \beta m}+\frac{C}{(d+2 \kappa)^{2}}
$$

with exponential accuracy. For the parameter values used in figure 3 , we note that $C$ is relatively small, while $C_{b}$ is relatively large. This allows us to simplify (43) further by neglecting the terms proportional to $C$, corresponding to the contributions from the tail of the distribution. Finally, neglecting terms of order $1 / m$, we obtain the leading-order approximation

$$
\frac{C_{b}-D}{\left(C_{b}-1\right) D} \sim \mathrm{e}^{-2 \kappa}
$$

and hence

$$
\nu \sim\left(\frac{C_{b}-D}{\left(C_{b}-1\right) D}\right)^{1 / m},
$$

which is valid when $D<C_{b}$. Figure 3 demonstrates that the simple approximations (41) and (45) are extremely accurate when $D>C_{b}$ and when $D<C_{b}$ respectively. We recall that realistic surfactants are likely to be modelled by even smaller values of $C$, in which case we can expect our approximations to be even better. 


\subsection{The governing equations}

After using (32) to scale the aggregate concentrations, we find that it is necessary also to rescale the time variable $\tau$ via

$$
\tau=m t
$$

to obtain a dominant balance in the governing equations. This result is in keeping with figures 5 and 6 , where the evolution to equilibrium was observed to occur over a large $\tau$ timescale. This longer timescale is a consequence of the large size of the typical aggregation number $m$, since larger aggregates will take longer to release all their monomers and break down entirely.

To obtain the continuum version of (29) we substitute (32) into (29a) and Taylor expand for small $\epsilon \equiv 1 / m$ to obtain

$$
\frac{\partial y}{\partial t}=\left(1-X_{1}(t)\right) \frac{\partial y}{\partial x}+\frac{\epsilon}{2}\left(1+X_{1}(t)\right) \frac{\partial^{2} y}{\partial x^{2}}-\epsilon \frac{\partial}{\partial x}\left(\frac{\bar{y}^{\prime}}{\bar{y}} y\right)
$$

where we neglect terms of order $\epsilon^{2}$, and prime denotes differentiation with respect to $x$. Similarly, substituting our continuum representation into (29b) and approximating the series as an integral provides

$$
X_{1}(t)=\frac{C_{b}}{\nu D}-\int_{0}^{\infty} x y(x, t) \mathrm{d} x,
$$

which enforces net conservation of surfactant and closes the system.

We note that $X_{1}$ tends towards 1 as the system approaches equilibrium, so that the first term on the right-hand side of ( $47 a)$ will eventually become comparable with the remaining terms on the right-hand side. We therefore retain terms of order $\epsilon$ in $(47 a)$ to ensure that the system is balanced at leading order during the entire evolution to equilibrium.

The initial condition for the problem is given by $(33 a)$, that is

$$
y(x, 0)=\frac{B_{0}}{\sqrt{\epsilon}} \exp \left(-\frac{\beta}{\epsilon}(x-1)^{2}\right)+C_{0} \exp (-d x) .
$$

In addition, the system (47) requires two boundary conditions. The first of these is obtained by writing the Becker-Döring equation (29a) with $n=2$ in terms of our continuous function $y$ and scaled time $t$ :

$$
\epsilon^{3} \frac{\partial y(2 \epsilon, t)}{\partial t}=X_{1}^{2}-\frac{y(\epsilon, t)}{\bar{y}(\epsilon)}-\epsilon^{2} X_{1} y(2 \epsilon, t)+\epsilon^{2} \frac{\bar{y}(2 \epsilon) y(2 \epsilon, t)}{\bar{y}(3 \epsilon)} .
$$

Again neglecting terms of $O\left(\epsilon^{2}\right)$, we obtain the boundary condition

$$
y(0, t)=X_{1}(t)^{2} \bar{y}(0),
$$


which implies that $y(0, t)$ instantaneously falls to $X_{1}(0)^{2} \bar{y}(0)$ for $t=0^{+}$. This indicates that dimers rapidly dissociate to replenish the depleted monomer, and is a consequence of the large difference between the concentrations of monomer and dimer in the solution. This rapid depletion occurs over a much shorter timescale on which $t=O\left(\epsilon^{3}\right)$ and all other aggregate concentrations remain fixed. The second boundary condition is provided by ensuring that we have a finite quantity of surfactant, which requires

$$
y(x, t) \rightarrow 0 \quad \text { as } x \rightarrow \infty .
$$

If we set $X_{1}=1$ and $\bar{y}=1$ then $(47 a)$ reduces to the standard diffusion equation. As discussed in the Introduction, there are many applications in which a ready supply of monomer ensures that $X_{1}$ remains effectively fixed at its equilibrium value. The assumption of constant monomer concentration may also be used to approximate the evolution of a system subject to very small dilutions, as in Aniansson and Wall [1, 2, 3].

\subsection{Comparison between discrete and continuum models}

In figure 7 we compare the evolution predicted by the Becker-Döring equations (29) with that predicted by our continuum model, using the distribution (15) with $b=10, \beta=0.2, C=0.5, d=1, C_{b}=10$ and $m=100$, upon a two-fold dilution. The parabolic equation $(47 a)$ is solved by the method of lines: a discretization in $x$ produces a system of nonlinear ordinary differential equations in $t$ which are solved using an implicit Euler scheme. The agreement between the evolutions predicted by the two models is excellent, except, as expected, in a small region as $x$ approaches zero where the discrete distribution rises to the monomer concentration (since $y$ is a continuous representation of the aggregate distribution for $n \geq 2$ ). The monomer concentration is also accurately captured by the continuum model, as illustrated in figure 8 .

In figure 9 we use $(47 a)$ to plot the evolution of the position of the maximum in the aggregate distribution. The distinct two-step behaviour of the re-equilibration is clearly apparent, with the position of the peak moving rapidly to the left to begin with, reaching a minimum at $t \approx 0.5$ before very slowly rising back to the new equilibrium value, at $x=\bar{\omega} \approx 0.980$ for this choice of parameters.

As discussed in $\S 2.5$, the rate at which the $\tau_{2}$ process occurs is dependent upon the concentration of aggregates in the intermediate region, which implies a vast separation between the timescales of the $\tau_{1}$ and $\tau_{2}$ processes when the relative concentration of intermediate aggregates is very low. This is shown in figure 10: as $C$ becomes very small, following the order-one $\tau_{1}$ 
process, the monomer concentration plateaus at a pseudo-equilibrium, which takes an exponentially long time to evolve towards the actual equilibrium (figure 11).

As discussed in the Introduction, many surfactants exhibit equilibrium distributions with extremely low concentrations of intermediate aggregates. However, it has been observed that such surfactant systems are able to re-equilibrate on a timescale much shorter than the Becker-Döring theory would suggest. For example, the surfactant $\mathrm{C}_{12} \mathrm{E}_{8}$ at a bulk concentration ten times the $\mathrm{CMC}$ has a typical concentration of intermediate aggregates $10^{-15}$ times lower than the concentration of proper micelles [10]. For such a system, the Becker-Döring theory predicts that the $\tau_{1}$ and $\tau_{2}$ processes will occur on timescales of the order of ms and hundreds of years respectively, while stopped-flow experiments suggest re-equilibration on the order of seconds [10]. It is thus evident that, while the Becker-Döring theory and our continuum model accurately describe the re-equilibration process for many surfactant systems, it fails to capture the physical mechanism of re-equilibration in all situations. This implies that there must be an alternative route that permits re-equilibration over sensible timescales for such surfactants and we will address this question in a follow-up paper.

\section{Conclusions}

In this paper, we examined the mechanism via which a micellar surfactant solution re-equilibrates following an order-one dilution. This extends the theory of Aniansson and Wall $[1,2,3]$ who consider only small variations in concentration. During re-equilibration, aggregates must release material to replenish the concentration of monomers back to its critical value, and we adopted the usual assumption that the breakdown or assembly of surfactant aggregates occurs via stepwise monomer loss or gain respectively. This leads to the set of coupled nonlinear ordinary differential equations known as the Becker-Döring equations. We considered a typical realistic system, in which the surfactant exists predominantly either in monomer form or as large aggregates (micelles) centred around an optimum aggregation number, with an intermediate separating region of much lower concentration.

For the majority of surfactant systems, the number of monomers that comprise a micelle is large. This permits the derivation of a continuum model, comprising a partial differential equation governing the evolution of aggregate concentrations coupled to an integral equation for the monomer concentration. Validation of the continuum model against the discrete Becker-Döring system showed excellent agreement while removing the complexity of tracking each species individually, which vastly simplifies numerical computation. 


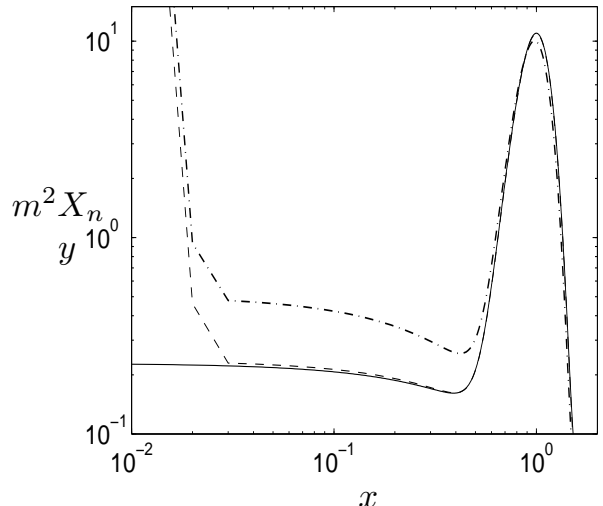

(a)

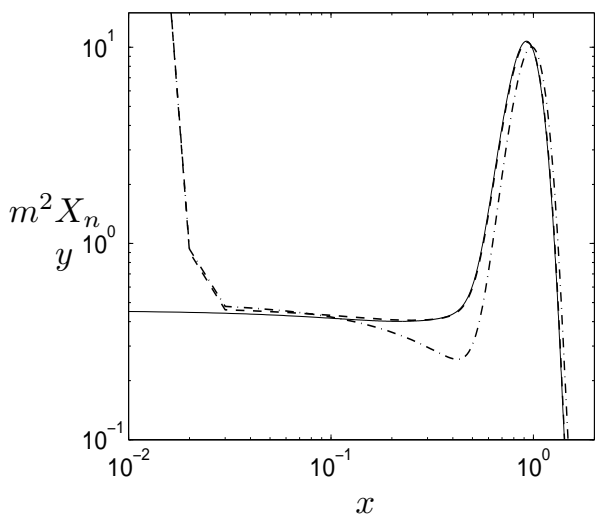

(c)

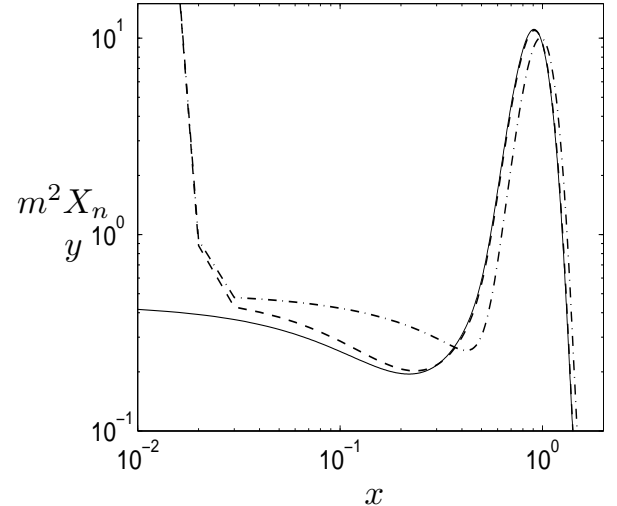

(b)

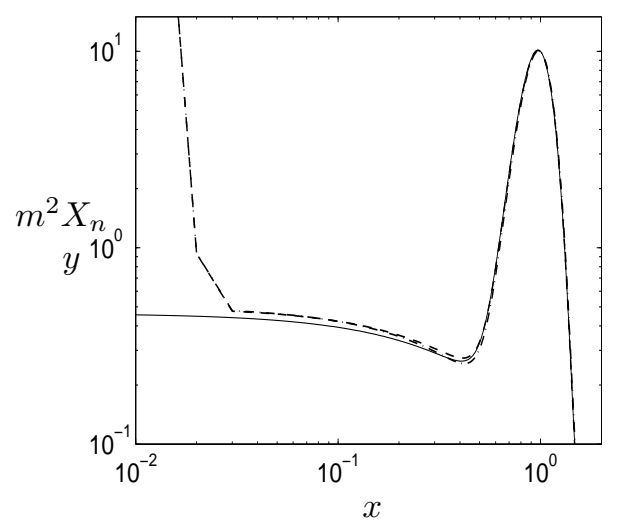

(d)

Figure 7: Comparison between the concentration profile evolutions predicted by the Becker-Döring equations (29) (dashed) and by the continuum model (47a) (solid) at times at (a) $t=0$, (b) $t=1$, (c) $t=5$, (d) $t=50$. The pre-dilution distribution (15) and continuum version (31) are used, with parameter values $b=10, \beta=0.2, C=0.5, d=1$, $m=100, C_{b}=10$ and $D=2$. The dot-dash line shows the equilibrium distribution. 


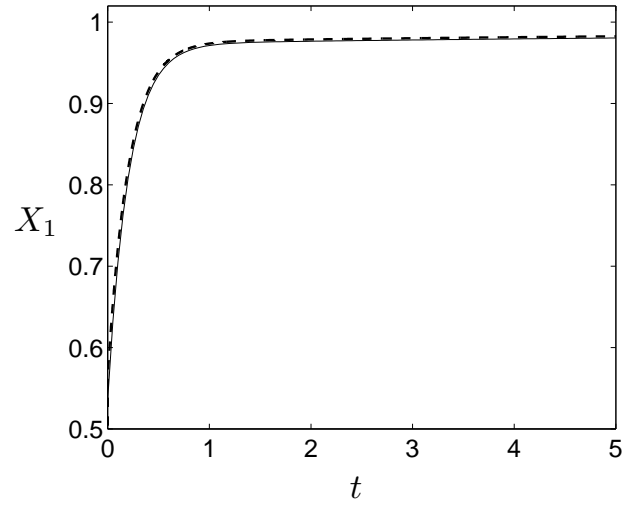

(a)

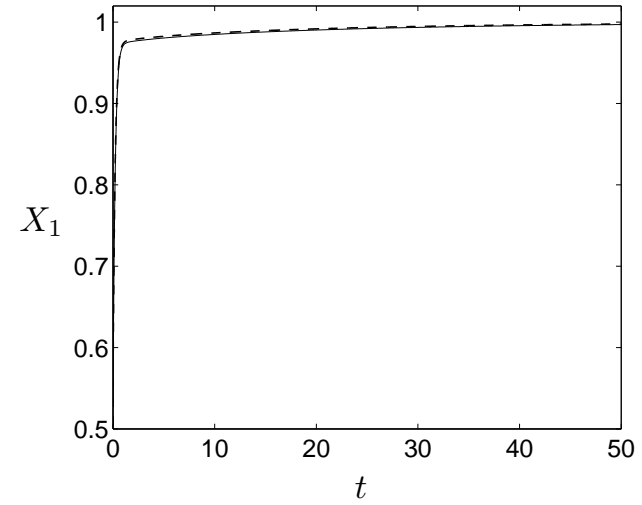

(b)

Figure 8: Comparison between the evolutions in the monomer concentration $X_{1}(t)$ predicted by the Becker-Döring equations (29) (dashed) and by the continuum model (47a) (solid) on (a) the $\tau_{1}$ timescale, (b) the $\tau_{2}$ timescale. The pre-dilution distribution (15) and continuum version (31) are used, with parameter values $\beta=0.2, C=0.5, d=1, m=100$, $C_{b}=10$ and $D=2$.
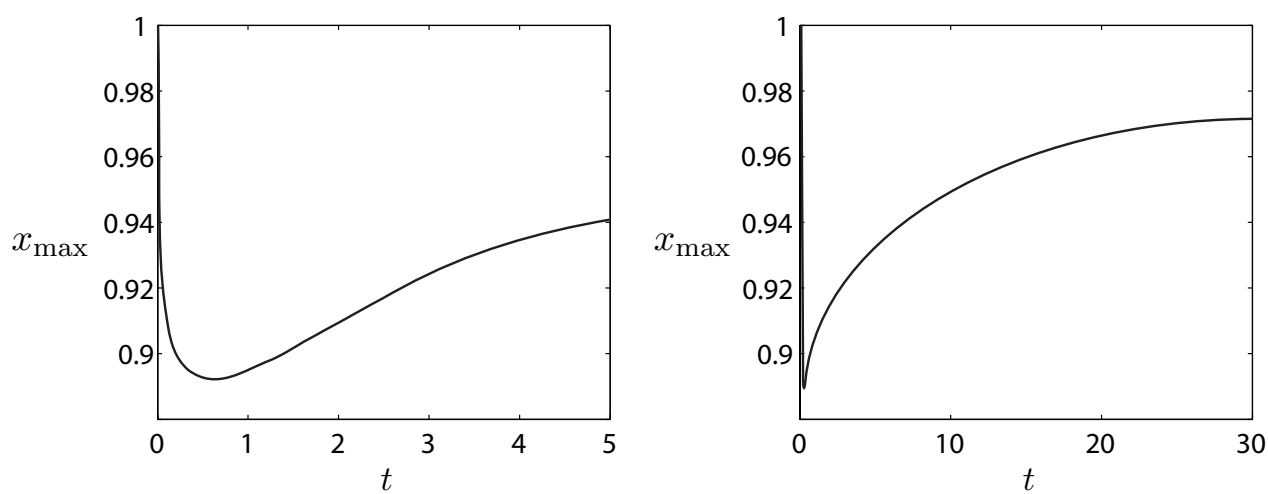

Figure 9: Evolution of the position of the maximum for the pre-dilution distribution (31), with $\beta=0.2, C=0.5, d=1, m=100, C_{b}=10$ and $D=2$, on (a) the $\tau_{1}$ timescale, (b) the $\tau_{2}$ timescale. 


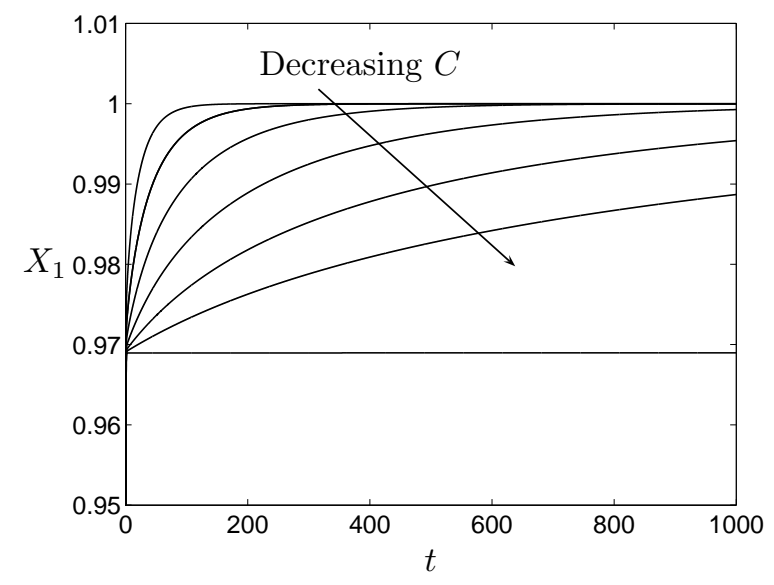

Figure 10: $\tau_{2}$ evolution of $X_{1}(t)$ with time predicted by the continuum model $(47 a)$. The pre-dilution distribution (15) and continuum version (31) are used, with parameter values $\beta=0.2, d=1, m=100, C_{b}=10$ and $D=2$, and we vary $C=\mathrm{e}^{-9}, \mathrm{e}^{-10}, \mathrm{e}^{-11}, \mathrm{e}^{-12}$, $\mathrm{e}^{-13}, \mathrm{e}^{-14}, 0$.

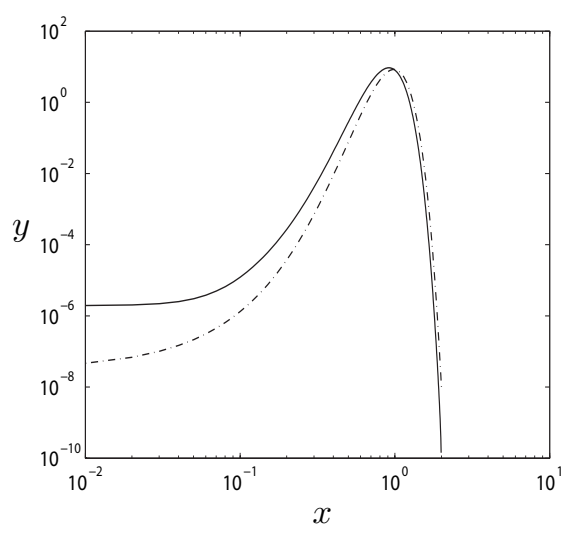

(a)

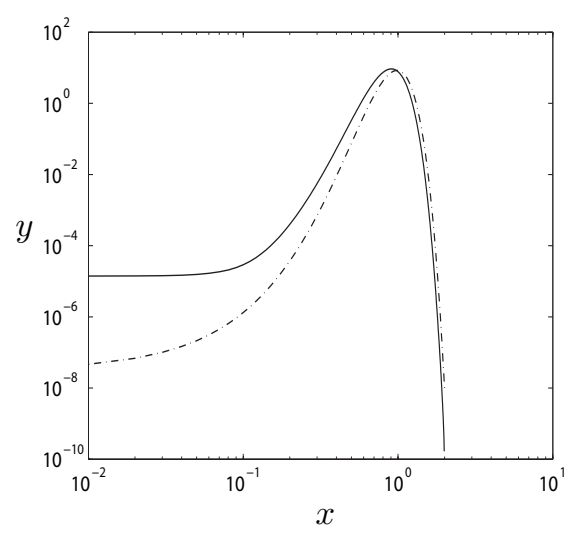

(b)

Figure 11: Concentration profile distribution at (a) $t=0.3$, (b) $t=10^{5}$ for a pre-dilution distribution (31) with parameter values $\beta=0.2, C=0, m=100, C_{b}=10$ and $D=2$. The profile effectively assumes a pseudo-equilibrium, taking an exponentially longer time to attain the actual system equilibrium. The dot-dash line shows the equilibrium distribution. 
The re-equilibration process was shown still to be characterized by two distinct stages, which are referred to as the $\tau_{1}$ and $\tau_{2}$ processes, in agreement with the behaviour observed following small dilutions $[1,2,3]$, and was validated against observations from stopped-flow experiments.

In the $\tau_{1}$ process, following dilution, the depleted monomer is replenished by the shedding of individual monomers from aggregates near micellar size, leading to a decrease in the dominant aggregate size. This occurs on a timescale of the order of microseconds. The $\tau_{2}$ process is characterized by the entire breakdown of some aggregates via stepwise monomer release. Here, some of the monomers released remain in this form to replenish the monomer to the equilibrium, while the remainder associate with some of the aggregates which have not dissociated. This has the simultaneous impact of increasing the dominant aggregate size while decreasing the total concentration of surfactant contained in aggregates.

The relative timescales of the $\tau_{1}$ and $\tau_{2}$ processes are related to the relative concentration of aggregates in the intermediate region. For typical surfactants, this is often many orders of magnitude lower than the concentration of proper micelles, leading to well-separated timescales. A key result of our analysis is that the separation between the time-scales of the $\tau_{1}$ and $\tau_{2}$ processes is exponentially large when the relative concentration of intermediate aggregates is low. In such cases, experimental observations indicate that the systems are able to re-equilibrate on a timescale very much shorter than this would suggest. While the Becker-Döring theory would predict that the $\tau_{1}$ and $\tau_{2}$ processes will occur on timescales of the order of ms and hundreds of years respectively, stopped-flow experiments indicate re-equilibration on the order of seconds [10].

We conclude that the standard theory fails to predict the correct reequilibration times for such surfactant systems. An alternative mechanism for re-equilibration that correctly explains the observed behaviour will be proposed in a follow-up to this paper.

This publication is based on work supported by Award No. KUK-C1-013-04, made by King Abdullah University of Science and Technology (KAUST) and by EPSRC grant EP/E019323. IMG gratefully acknowledges helpful discussions with Dr P. J. Dellar, Professor S. D. Howison and Professor J. R. Ockendon. SLW is grateful to the EPSRC for funding in the form of an Advanced Research Fellowship. 


\section{References}

[1] Aniansson, E. A. G., Wall, S. N., 1974. On the kinetics of step-wise micelle association. J. Phys. Chem. 78, 1024-1030.

[2] Aniansson, E. A. G., Wall, S. N., 1975. Kinetics of step-wise micelle association and dissociation. Correction and improvement. J. Phys. Chem. $79,857-858$.

[3] Aniansson, E. A. G., Wall, S. N., Almgren, M., Hoffmann, H., Kielmann, I., Ulbricht, W., Zana, R., Lang, J., Tondre, C., 1976. Theory of the kinetics of micellar equilibria and quantitative interpretation of chemical relaxation studies of micellar solutions of ionic surfactants. J. Phys. Chem. 80 (9), 905-922.

[4] Band, L. R., Cummings, L. J., Waters, S. L., Wattis, J. A., 2009. Modelling crystal aggregation and deposition in the catheterised lower urinary tract. J. Math. Biol. 59, 809-840.

[5] Becker, R., Döring, W., 1935. Kinetische behandlung der Keimbildung in ubersattigten dampfern. Ann. Phys. 24, 719-752.

[6] Billingham, J., 2000. On modelling the formation of micelles in the presence of a slow influx of monomer. Q. J. Mech. Appl. Math. 53 (2), 285.

[7] Billingham, J., Coveney, P. V., 1994. Kinetics of self-replicating micelles. J. Chem. Soc. 90 (13), 1953-1959.

[8] Chertkov, E. V., Saidov, A. A., Khabibullaev, P. K., 2000. On the kinetics of the micelle forming systems. Colloids and Surfaces A 168 (3), $185-191$.

[9] Colegate, D. M., 2008. Private communication.

[10] Colegate, D. M., 2009. Structure-kinetics relationships in micellar solutions of nonionic surfactants. Ph.D. thesis, Durham University.

[11] Corkill, J. M., Goodman, J. F., Harrold, S. P., 1964. Thermodynamics of micellization of non-ionic detergents. Trans. Faraday Soc. 60, 202-207.

[12] Coveney, P. V., Wattis, J. A. D., 1996. Analysis of a generalized BeckerDöring model of self-reproducing micelles. Proc. Math. Physi. Eng. Sci., 2079-2102. 
[13] de Moraes, J. N. B., Figueiredo, W., 2001. Dynamical study of a micellar system. Physica Status Solidi 187 (1), 57-62.

[14] Frindi, M., Michels, B., 1992. Ultrasonic absorption studies of surfactant exchange between micelles and bulk phase in aqueous micellar solutions of nonionic surfactants with a short Alkyl chain. 2. $\mathrm{C}_{6} \mathrm{E}_{3}, \mathrm{C}_{6} \mathrm{E}_{5}, \mathrm{C}_{8} \mathrm{E}_{4}$, and $\mathrm{C}_{8} \mathrm{E}_{8}$. J. Phys. Chem. 96, 6095-6102.

[15] Guy, R. D., Fogelson, A. L., Keener, J. P., 2007. Modeling fibrin gel formation in a shear flow. Math. Medicine Biol. 24, 111-130.

[16] Jorge, M., 2008. Molecular dynamics simulation of self-assembly of nDecyltrimethylammonium Bromide micelles. Langmuir 24 (11), 57145725 .

[17] Kato, S., Nomura, H., Honda, H., Zielinski, R., Ikeda, S., 1988. Ultrasonic relaxation study of the exchange process of surfactant monomer with micelle in aqueous solutions of alkyltrimethylammonium bromides. J. Phys. Chem. 92 (8), 2305-2310.

[18] Kuni, F. M., Grinin, A. P., Shchekin, A. K., Rusanov, A. I., 2001. Thermodynamic and kinetic foundations of the micellization theory: 4 . Kinetics of establishment of equilibrium in a micellar solution. Colloid Journal 63 (2), 197-204.

[19] Kuni, F. M., Grinin, A. P., Shekin, A. K., Rusanov, A. I., 2000. Thermodynamic and kinetic foundations of the micellization theory: 3. Initial stages of micellization. Colloid Journal 62 (4), 451-456.

[20] Kuni, F. M., Rusanov, A. I., Grinin, A. P., Shchekin, A. K., 2001. Thermodynamic and kinetic foundations of the micellization theory: 5 . Hierarchy of kinetic times. Colloid Journal 63 (6), 723-730.

[21] Kuni, F. M., Shchekin, A. K., Grinin, A. P., Rusanov, A. I., 2000. Thermodynamic and kinetic foundations of the micellization theory: 2 . Direct and reverse fluxes of molecular aggregates over the activation barrier of micellization. Colloid Journal 62 (2), 172-178.

[22] Lang, J., Tondre, C., Zana, R., Bauer, R., Hoffmann, H., Ulbricht, W., 1975. Chemical relaxation studies of micellar equilibriums. J. Phys. Chem. 79 (3), 276-283.

[23] Leung, R., Shah, D. O., 1986. Dynamic properties of micellar solutions. I: Effects of short-chain alcohols and polymers on micellar stability. J. Colloid Int. Sci. 113 (2), 484-499. 
[24] Malliaris, A., Lang, J., Zana, R., 1986. Dynamics of micellar solution of ionic surfactants by fluorescence probing. J. Phys. Chem. 90 (4), 655660 .

[25] Mohan, G., Kopelevich, D. I., 2008. A multiscale model for kinetics of formation and disintegration of spherical micelles. J. Chem. Phys. 128, 044905 .

[26] Nagarajan, R., Ruckenstein, E., 1979. Aggregation of amphiphiles as micelles or vesicles in aqueous media. J. Colloid Int. Sci. 71 (5), 580604.

[27] Nagarajan, R., Ruckenstein, E., 1991. Theory of surfactant selfassembly: a predictive molecular thermodynamic approach. Langmuir 7, 2934-2969.

[28] Patist, A., Kanicky, J. R., Shukla, P. K., Shah, D. O., 2002. Importance of micellar kinetics in relation to technological processes. J. Colloid Int. Sci. 245 (1), 1-15.

[29] Penrose, O., Lebowitz, J. L., 1987. Towards a rigorous molecular theory of metastability. Fluctuation Phenomena 7, 293-340.

[30] Puvvada, S., Blankschtein, D., 1990. Molecular-thermodynamic approach to predict micellization, phase behavior and phase separation of micellar solutions. I. Application to nonionic surfactants. J. Chem. Phys. 92,3710 .

[31] Richardson, G., Cummings, L. J., Harris, H. J., O’Shea, P., 2007. Toward a mathematical model of the assembly and disassembly of membrane microdomains: comparison with experimental models. Biophysical Journal 92, 4145-4156.

[32] Rusanov, A. I., Kuni, F. M., Shchekin, A. K., 2000. Thermodynamic and kinetic foundations of the micellization theory: 1. General aspects. Colloid Journal 62 (2), 167-171.

[33] Smoluchowski, V., 1917. Mathematical Theory of the Kinetics of the Coagulation of Colloidal Particle. Phys. Chem 92, 129-168.

[34] Teubner, M., 1979. Theory of ultrasonic-adsorption in micellar solutions. J. Phys. Chem. 83, 2917-2920. 
[35] Ueno, M., Takasawa, Y., Miyashige, H., Tabata, Y., Meguro, K., 1981. Effects of alkyl chain length on surface and micellar properties of octaethyleneglycol-n alkyl ethers. Colloid \& Polymer Science 259 (7), 761-766.

[36] Wattis, J., King, J., 1998. Asymptotic solutions of the Becker-Döring equations. J. Phys. A 31, 7169.

[37] Wattis, J. A. D., 2006. An introduction to mathematical models of coagulation-fragmentation processes: a discrete deterministic mean-field approach. Physica D: Nonlinear Phenomena 222 (1-2), 1-20.

[38] Yasunaga, T., Takeda, K., Harada, S., 1973. Kinetic study of Sodium Dodecyl Sulfate micelle dissociation by a stopped-flow method. J. Colloid Int. Sci. 42, 457-463.

[39] Yushmanov, P. V., Furo, I., Stilbs, P., 2006. Micellar kinetics of a fluorosurfactant through stopped-flow NMR. Langmuir 22 (5), 2002-2004. 



\section{RECENT REPORTS}

53/10 Liquid Crystal Theory and Modelling Discussion Meeting Majumdar

Mottram

54/10 Modeling the growth of multicellular cancer spheroids in a bioengi- Loessner neered 3D microenvironment and their treatment with an anti- Flegg cancer drug

Byrne

Hall

Moroney

Clements

Hutmacher

McElwain

55/10 Scalar Z, ZK, KZK, and KP equations for shear waves in incompressible solids

Destrade

Goriely

Saccomandi

56/10 The Influence of Bioreactor Geometry and the Mechanical Environment on Engineered Tissues

Osborne

ODea

Whiteley

Byrne

Waters

$57 / 10$ A numerical guide to the solution of the bidomain equations of cardiac electrophysiology

Pathmanathan

Bernabeu

Bordas

Cooper

Garny

Pitt-Francis

Whiteley

Gavaghan

58/10 Particle-scale structure in frozen colloidal suspensions from small

Spannuth angle X-ray scattering

Mochrie

Peppin

Wettlaufer

59/10 Spin coating of an evaporating polymer solution Munch

Please

Wagner

60/10 Stochastic synchronization of neuronal populations with intrinsic and extrinsic noise

Bressloff

Lai

61/10 Metastable states and quasicycles in a stochastic Wilson-Cowan

Bressloff model of neuronal population dynamics

62/10 Adsorption and desorption dynamics of citric acid anions in soil

Oburger

Leitner

Jones

Zygalakis

Schnepf

Roose

63/10 A dual porosity model of nutrient uptake by root hairs soil

Zygalakis

Kirk

Jones

Roose

Wissuwa 
02/11 Self-diffusion in remodelling and growth Epstein

03/11 Spontaneous rotational inversion in Phycomyces Goriely

Tabor

04/11 From individual to collective behaviour of coupled velocity jump Erban processes: a locust example

Haskovec

05/11 Solving Eigenvalue problems on curved surfaces using the closest point method

MacDonald

Brandman

Ruuth

06/11 A numerical methodology for the Painleve equations

Fornberg

Weideman

07/11 Strong stability preserving two-step Runke-Kutta methods

Ketcheson

Gotlieb

MacDonald

08/11 Hysteresis and Post Walrasian Economics

Cross

McNamara

Kalachev

Pokrovskii

09/11 A locally adaptive time-stepping algorithm for petroleum reservoir

McNamara simulations

Bowen

Dellar

Copies of these, and any other OCCAM reports can be obtained from:

Oxford Centre for Collaborative Applied Mathematics Mathematical Institute

24 - 29 St Giles'

Oxford

OX1 3LB

England

www.maths.ox.ac.uk/occam 